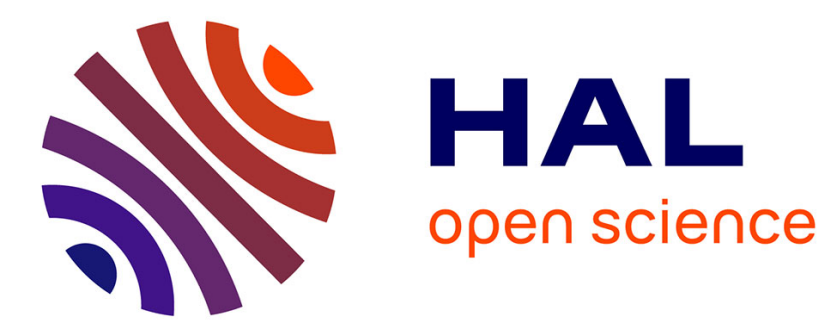

\title{
Nature et origine des concentrations de minéraux lourds sur les grèves de Roscoff (Finistère, France). Sources proximales et apports distaux sur une côte contraposée Louis Chauris
}

\section{- To cite this version: \\ Louis Chauris. Nature et origine des concentrations de minéraux lourds sur les grèves de Roscoff (Finistère, France). Sources proximales et apports distaux sur une côte contraposée. Norois, 1986, 33 (130), pp.161-178. 10.3406/noroi.1986.4302 . insu-01860999}

\section{HAL Id: insu-01860999 \\ https://hal-insu.archives-ouvertes.fr/insu-01860999}

Submitted on 17 Feb 2021

HAL is a multi-disciplinary open access archive for the deposit and dissemination of scientific research documents, whether they are published or not. The documents may come from teaching and research institutions in France or abroad, or from public or private research centers.
L'archive ouverte pluridisciplinaire HAL, est destinée au dépôt et à la diffusion de documents scientifiques de niveau recherche, publiés ou non, émanant des établissements d'enseignement et de recherche français ou étrangers, des laboratoires publics ou privés.

\section{(ㅇ)(1) $\$$}

Distributed under a Creative Commons Attribution - NonCommercial - NoDerivatives| 4.0 


\section{Nature et origine des concentrations de minéraux lourds sur les} grèves de Roscoff (Finistère, France). Sources proximales et apports distaux sur une côte contraposée

\section{Louis Chauris}

\section{Citer ce document / Cite this document :}

Chauris Louis. Nature et origine des concentrations de minéraux lourds sur les grèves de Roscoff (Finistère, France). Sources proximales et apports distaux sur une côte contraposée . In: Norois, n¹30, Avril-Juin 1986. pp. 161-178;

doi : https://doi.org/10.3406/noroi.1986.4302

https://www.persee.fr/doc/noroi_0029-182x_1986_num_130_1_4302

Fichier pdf généré le 10/11/2019 


\title{
Résumé
}

Soixante échantillons de sables lourds ont été prélevés dans les petits placers littoraux de la presqu'île de Roscoff (Massif armoricain). Les facteurs hydrodynamiques sont commandés par la grande amplitude des marées et surtout par les houles du secteur ouest. Les dépôts présentent de fortes variations de teneurs, dues à leur localisation par rapport aux roches-mères et surtout aux différences dans l'énergie des vagues. Le spectre minéralogique est dominé par l'amphibole (34 \%), le grenat (26 $\%)$, l'ilmênite $(17 \%)$ et la tourmaline (10\%). Viennent ensuite sphène, staurotide et épidote ; puis magnetite, monazite, zircon et rutile ; les autres minéraux ne sont présents qu'à l'état de traces. La comparaison des cartes de répartition des minéraux lourds souligne la complexité des transits qui peuvent même avoir lieu en sens opposés. De nombreux minéraux dérivent sans relais des altérites voisines (sources primaires proximales). D'autres minéraux proviennent du remaniement des sables dunaires qui constituent des relais secondaires (les sources primaires des minéraux lourds sont alors distales). En règle générale, les concentrations offrent un net indice de jeunesse ; le spectre d'ensemble est typiquement immature.

\begin{abstract}
Nature and origin of heavy mineral concentrations along the coast of roscoff (Finistere, France) proximal and distal sources on a contraposed shoreline - Sixty samples of heavy sands were collected from coastal micro-placers exposed along the shore of the Roscoff peninsula (Armorican Massif), where rocky coves and dune sands alternate. A large amplitude of the tides and, even more, strong western swells are the main hydrodynamical factors. The samples, preferentially taken on the uppermost part of the strand, show important variations in heavy mineral percentages, owing to their location with regard to the source-rocks, to the contamination by biogenic sands and especially to strong differences in wave energy. The mineralogical composition is dominated by amphibole (34 \%), garnet (26\%), ilmenite (17\%) and tourmaline (10\%). Then sphene, staurolite and epidote come ; and then magnetite, monazite, zircon and rutile; the other minerals are only present in traces. The comparison of the maps emphasizes the complexity in the transfers which can occur sometimes in opposite directions. North-south and east-west transportations can be superposed to a general westeast drift. The two principal types of coast control the origin of the concentrations- Numerous minerals come without relay from adjoining weathered rocks (proximal primary sources) ; some mineral (tracers) allow to outline haloes of dispersion from source-rocks. Other minerals come from reworking and sorting of dune sands which form secondary relays (the primary origins of heavy mineral are then distal sources). As a general rule, the concentrations present a strong index of youthfulness with, as a whole, an immature composition.
\end{abstract}


Norois, 1968, Poitiers, t. 33, $\mathrm{n}^{\circ} 130$, p. 161-178.

\title{
Nature et origine des concentrations de minéraux lourds sur les grèves de Roscoff (Finistère, France). Sources proximales et apports distaux sur une côte contraposée
}

\author{
par Louis CHAURIS \\ Département des Sciences de la Terre. \\ G.l.S. "Océanologie et Géodynamique 410012 " \\ Université de Bretagne Occidentale - 29287 Brest.
}

A la mémoire de Jacques Bourcart

\begin{abstract}
RESUME
Soixante échantillons de sables lourds ont été prélevés dans les petits placers littoraux de la presqu'île de Roscoff (Massif armoricain). Les facteurs hydrodynamiques sont commandés par la grande amplitude des marées et surtout par les houles du secteur ouest. Les dépôts présentent de fortes variations de teneurs, dues à leur localisation par rapport aux roches-mères et surtout aux différences dans l'énergie des vagues. Le spectre minéralogique est dominé par l'amphibole $(34 \%)$, le grenat $(26 \%)$, l'ilménite $(17 \%)$ et la tourmaline $(10 \%)$. Viennent ensuite sphène, staurotide et épidote; puis magnétite. monazite, zircon et rutile ; les autres minéraux ne sont présents qu'à l'état de traces. La comparaison des carles de répartition des minéraux lourds souligne la complexité des transits qui peuvent même avoir lieu en sens opposés. De nombreux minéraux dérivent sans relais des altérites voisines (sources primaires proximales). D'autres minéraux proviennent du remaniement des sables dunaires qui constituent des relais secondaires (les sources primaires des minéraux lourds sont alors distales). En règle générale, les concentrations offrent un net indice de jeunesse; le spectre d'ensemble est typiquement immature.
\end{abstract} ABSTRACT
NATURE AND ORIGIN OF HEAVY MINERAL CONCENTRATIONS
ALONG THE COAST OF ROSCOFF (FINISTERE, FRANCE)
PROXIMAL AND DISTAL SOURCES ON A CONTRAPOSED SHORELINE
Sixty samples of heavy sands were collected from coastal micro-placers exposed
along the shore of the Roscoff peninsula (Armorican Massif), where rocky coves

Mots-clés : Minéraux lourds. Placers littoraux. Sources proximales et distales. Roscoff. Massif armoricain.

Key-words : Heavy minerals. Beach placers. Proximal and distal sources. Roscoff. Armorican massif. 
and dune sands alternate. A large amplitude of the tides and, even more, strong western swells are the main hydrodynamical factors. The samples, preferentially taken on the uppermost part of the strand, show important variations in heavy mineral percentages, owing to their location with regard to the source-rocks, to the contamination by biogenic sands and especially to strong differences in wave energy. The mineralogical composition is dominated by amphibole (34\%), garnet $(26 \%)$, ilmenite $(17 \%)$ and tourmaline $(10 \%)$. Then sphene, staurolite and epidote come ; and then magnetite, monazite, zircon and rutile; the other minerals are only present in traces. The comparison of the maps emphasizes the complexity in the transfers which can occur sometimes in opposite directions. North-south and east-west transportations can be superposed to a general westeast drift. The two principal types of coast control the origin of the concentrations. Numerous minerals come without relay from adjoining weathered rocks (proximal primary sources); some mineral (tracers) allow to outline haloes of dispersion from source-rocks. Other minerals come from reworking and sorting of dune sands which form secondary relays (the primary origins of heavy mineral are then distal sources). As a general rule, the concentrations present a strong index of youthfulness with, as a whole, an immature composition.

\section{INTRODUCTION}

Dans un article précédent de Norois (Chauris, 1984), nous avons montré l'intérêt d'une étude des concentrations de minéraux lourds (M.L.) sur les plages de la baie de Morlaix (Finistère), pour la solution d'un problème morphogénétique : celui de l'origine des sables qui forment ces plages. Il était apparu que les sources locales (origine proximale) étaient préprépondérantes en cette baie relativement abritée des houles du large. Sur un ensemble de plages du Nord-Finistère, la même méthode a conduit à la conclusion (Chauris, 1982) d'une grande variété de cortèges minéralogiques, les contrastes apparaissant comme le reflet de l'arrièrepays. Dans la presqu'île du Petit-Trégor, située à l'est de la baie de Morlaix, des apports occidentaux limités (source distale) se superposent aux sources proximales qui se sont avérées dominantes (Chauris, 1985).

Le présent article, qui s'insère dans la même perspective de recherche morphogénétique, par les minéraux lourds, des sources proximales et/ou distales des sables des plages armoricaines, repose sur l'examen détaillé du district situé à l'ouest de la baie de Morlaix : la presqu'île de Roscoff, prolongée au nord par l'île de Batz (fig. 1). La région roscovite est beaucoup plus exposée aux houles occidentales que les districts précités. La morphologie littorale répond à la définition de " côte contraposée " (Guilcher, 1954). Un remblaiement meuble d'épaisseur variable (arènes, coulées de head, placages de limons, pellicules de sables dunaires) a particllement masqué la topographie différenciée du substratum résistant ; l'action de la mer consiste principalement à exhumer les chaos rocheux préexistants (ou monadnocks) de leur couverture récente.

Les observations sur le terrain ont été poursuivies de 1981 à 1985, au cours de diverses saisons. Les prélèvements, de l'ordre de quelques centaines de grammes par échantillon, ont été effectués par raclage à la pelle des placages minéralisés qui correspondent à de petits placers littoraux (cf. Burns, 1975 ; Cronan, 1980; Macdonald, 1983). Au total, 60 échantillons ont été analysés par la méthode décrite dans les ouvrages 


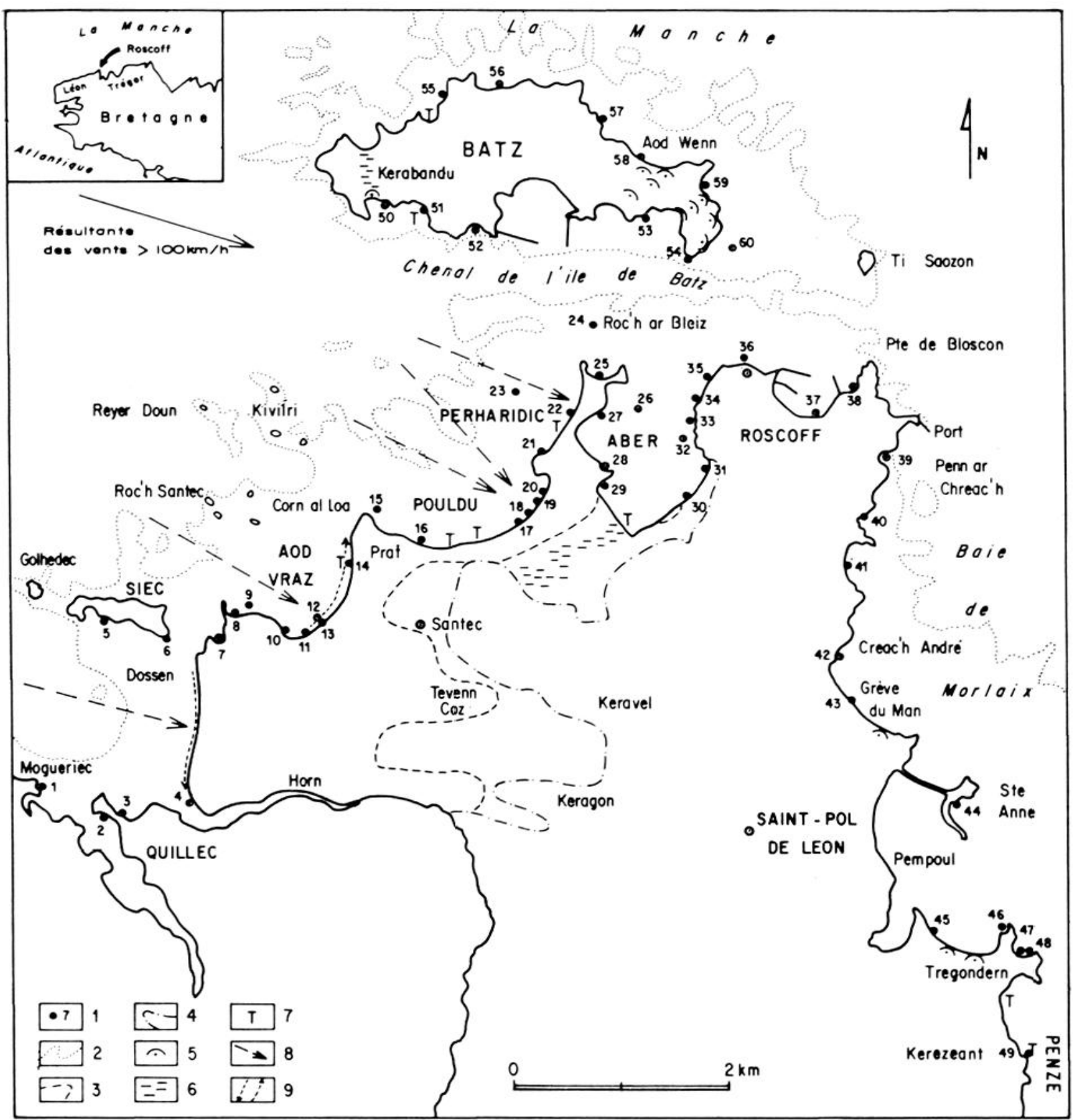

Fig. 1 - 1 - Localisation des échantillons. 2 - Limite des plus basses mers. 3 Limite orientalc des sables dunaires de Santec. 4. Limite orientale de la pellicule sableuse. (Limites 3 et 4 non cartographiées au sud de l'Horn. 5 - Autres dunes. 6 - Zones humides. 7 - Tourbières littorales. 8 - Direction principale de la houle sur les grandes plages. 9 - Dérive littorale en sens opposé à Aod Vraz et au Dossen.

FIG. 1 - 1 - Localization of the samples. 2 - Boundary of the low-tide. 3 - Eastern boundary of the Santec dune sands. 4 - Eastern boundary of the sandy sheet. (Boundaries 3 and 4 not mapped southern Horn). 5 - Other dunes. 6 - Wetlands. 7. Coastal peat-bogs. 8 - Main direction of swell on large beaches. 9 - Coastal drift in an opposite direction at Aod Vraz and at Dossen. 
de Guigues et al. (1969) et Devismes (1978). Le pourcentage des M.L. ( $d>2,9$ ) par rapport au sable brut est donné en poids; le pourcentage des différents M.L. est également donné en poids.

Les études antérieures sur les M.L. de la région de Roscoff sont, le plus souvent, restées ponctuelles (Duplaix, 1946 ; Dupeuble, 1964 ; Duplaix et al., 1968). Seules les recherches de Francis-Bœuf et al. (1949) sur l'Aber de Roscoff ont été entreprises à une échelle détaillée, mais il est regrettable que les minéraux opaques (ilménite, magnétite) n'aient pas été déterminés.

\section{I. - Le caDre NATUREL.}

La région de Roscoff est situće à l'extrémité nord-est du Pays de Léon. Le socle cristallophyllien (probablement cadomien) est constitué essentiellement par des gneiss de types varićs et, plus localement, par des micaschistes à grenat et staurotide ou des amphibolites à hornblende verte. Ces formations sont recoupées par le pluton hercynien polyphasé de Roscoff : diorites à hornblende et sphène ; granites gris ; leucogranites à tourmaline et grenat, pegmatites à grenat, tourmaline, parfois apatite ou béryl. A l'extrémité orientale de la région affleure le granite rose tardihercynien de la baie de Morlaix (Barrois, 1909 ; Sandrea, 1958 ; Chauris, 1975 ; Chauris et al., 1978a et b, 1981).

Le substratum rocheux, souvent arénisé, est fréquemment masqué par les recouvrements quaternaires (Bourcart, 1948) : plages «bas-normaniennes "(Eemien) ; coulées de solifluxion (head) et limons würmiens (Wechselien) ; tourbes (la plus récente, à Jugan en Santec est datée à $2330 \pm 105$ B.P. (Morzadec-Kerfourn, 1974); enfin, sables dunaires dont la mise en place s'est poursuivic depuis l'Age du Fer jusqu'au Xvill siècle (Hallégouët, 1978). Sur les estrans, le revêtement sableux actuel est souvent très mince : limons et tourbes affleurent parfois directement (Aber, Pouldu...). Les dépôts de sable s'épaississent localement sur la partie haute de l'estran dans la zone de passage aux dunes ou sur leur partie basse sous forme de bancs allongés (ouest de Roc'h ar Bleiz au nord de Perharidic...).

La morphologie littorale présente trois types principaux. (a) Les rivages rocheux (baie de Morlaix, île de Batz, île de Siec...) constitués par des monadnocks granitiques qui isolent de petites criques (Bourcart, 1948). Les mêmes formes de relief résiduel s'observent dans le bas-pays émergé (en avant de la falaise-morte qui marque la limite de la mer éemienne), sur l'estran et dans la zone infra-tidale. (b) Les côtes dunaires bordées de grandes plages (Dossen, Aod, Vraz, Pouldu, Perharidic...), en voie d'attaque, sauf à l'extrémité méridionale du Dossen où s'édifie un système de crêtes dunifiées du type Darss (Castro, 1981 ; Hallégouët, 1981). La situation des petites rias de l'Horn et du Quilliec à l'cxtrémité méridionale des dunes de Santec explique les bancs de sables qui encombrent leur embouchure. (c) L'anse de l'Aber est un ancien marais littoral, jadis formé à l'abri du cordon dunaire du Pouldu-Perharidic et à présent envahi par la mer qui remanie à chaque marée la mince pellicule de sables en provenance des dunes voisines. 
Les facteurs hydrodynamiques sont commandés : (a) par l'amplitude des marées (jusqu'à plus de $9 \mathrm{~m}$ ) qui induit de forts courants alternatifs (chenal de l'île de Batz, avec flot d'ouest en est et jusant d'est en ouest) et qui laisse à nu un large estran $(1,2 \mathrm{~km}$ au droit d'Aod Vraz; $2,4 \mathrm{~km}$ au droit de l'Aber); (b) par la force des vagues liée à un fetch variable selon l'orientation de la côte. La rose des vents (station de l'île de Batz) souligne la nette prédominance des vents d'ouest (secteur $\mathrm{NW}$ à $\mathrm{SW}=$ $52,9 \%$, Conversey, 1962). La résultante des vents supérieurs à $100 \mathrm{~km} / \mathrm{h}$ est orientée WNW-ESE (Castro, 1981).

Les conséquences de l'influence prépondérante des vents de secteur ouest sont nombreuses. (a) A l'île de Batz, contraste entre la côte occidentale, essentiellement rocheuse et dénudée, et la côte orientale, prolongée en zone d'abri par un immense estran sablcux ; différence sensible dans l'émoussé des galets entre les parties ouest et est de l'île (respectivement 386 et 295, Conversey, 1962. (b) A Santec, l'examen des cartes anciennes, du cadastre et des photographies aćriennes permet de chiffrer le recul de la côte (Croguennoc, 1981) : au Pouldu, entre 1846 et 1970, recul de $65 \mathrm{~m}$ dans la portion de la plage exposéc au NW (propagation facile de la houle dans les couloirs sableux) et de $35 \mathrm{~m}$ dans la portion plus abritée de la plage orientée au nord ; dans la partie centrale des dunes du Dossen exposées à l'ouest, l'ćrosion a été de $50 \mathrm{~m}$ en 30 ans. Les reculs spectaculaires sont liés à la coïncidence de fortes tempêtes avec de grandes marées : au Dossen, début novembre 1967, par marée de coefficient 112, accompagnée de vent de $115 \mathrm{~km} / \mathrm{h}, 8$ à 10 mètres de dunes ont été emportés. (c) Au cours des derniers millénaires, de tels reculs ont entraîné des modifications totales du trait de côte : insularité de l'île de Batz, envahissement du marais littoral de l'Aber.... Quelques pointes rocheuses résistent encore (ouest de Siec, de Batz,...), mais d'anciens promontoires sont réduits à l'état de récifs isolés (Roc'h Santec, Reyer Doun...).

Les mouvements des sédiments sur les estrans et dans les zones immergées voisines sont trìs complexes ct, selon les points, souvent de sens opposé. Sur la plage d'Aod Vraz, la dérive littorale se propage du sud au nord, tandis que sur la plage du Dossen, elle progresse du nord au sud. Au nord de Perharidic, les récifs abritent des queues de comète aux directions opposées (Conversey, 1962). Au large de Roscoff, le transit général paraît s'effectuer d'ouest en est (Le Gorgeu et al., 1964), mais en baie de Morlaix, des déplacements se font localement (dune hydaulique de la Pierre Noire) en direction de l'ouest (Auffret et al., 1974).

Les minéraux lourds des accumulations sablcuses sont soumis à ces différentes forces. Certaines espèces jouent le róle de traceurs et contribuent ainsi à une meilleure compréhension des processus hydrodynamiques.

\section{II. - LeS ACCUMUlations de MinÉRAUX LOURDS.}

Dans la région de Roscoff, les M.L. se concentrent dans des sites variés. (a) Sur les parties moyennes et basses de l'estran, les dépôts sont toujours de faible intensité. Ils présentent diverses modalités : sur les 
grandes plages sableuses (partie nord-est du Pouldu), succession de bandes parallèles au rivage; sur les bancs de sables (dunes hydrauliques) accidentés de grosses rides dissymétriques, vers la partie supérieure du versant à forte pente (ouest de Roc'h ar Blciz ; sud-est de l'île de Batz,...) ; dans le lit des ruisselets liés au jusant, divagant sur l'estran à basse mer, etc... (b) Sur la partie haute des plages, les accumulations sont plus importantes. Les dépôts se réduisent souvent à des placages superficiels d'épaisseur millimétrique à centimétrique, de faible extension longitudinale (quelques mètres à quelques dizaines de mètres). Sur la grève du Man et dans la partie nord-est de la plage du Pouldu, des concentrations sont périodiquement visibles sur plusieurs centaines de mètres, mais avec des tencurs relativement faibles (respectivement 24 et $35 \%$ ). Près de la Penzé, aux environs de Kerezeant, des accumulations de plusieurs centimètres de puissance s'ćchelonnent d'une manière discontinue sur plus de 800 mètres. C'est également ici que se rencontrent les teneurs les plus élevées en M.L. $(84,6 \%$ à Kerezeant). Le district de la Penzé mis à part, seuls 5 prélèvements ont présenté des teneurs comprises entre 50 et $60 \%$. Les autres échantillons offrent des teneurs nettement plus faibles, parfois inférieures à $10 \%$. Les dépôts sont généralements constitués de plusieurs couches parallèles de teinte rougeâtre à noirâtre, qui alternent avec des sables stériles blanchâtres. Dans quelques cas, les accumulations de sables lourds reposent directement sur le limon (près du Ruguel dans l'Aber, localement à Aod Vraz...).

Les causes des variations de teneurs sont multiples. La proximité d'une roche-mère riche en M.L. entraîne généralement de fortes concentrations : ainsi s'expliquent les teneurs élevées des placers de la Penzé qui dérivent des amphibolites. Plus souvent, l'opposition entre zone riche et zone pauvre est fonction des variations dans l'énergie disponible pour un triage sélectif des M.L., avec concentration préférentielle dans les zones de hautc ćncrgic. A l'ouest du Pouldu, il apparaît un fort contraste de teneurs entre l'échantillon 15 , prélevé à quelque distance du rivage $(2,4 \%$ de M.L.) et l'échantillon 16, prélevé sur le haut de l'estran ( $25 \%$ de M.L.). Dans la partie est de l'Aber, le contraste est net entre l'échantillon 32, recueilli assez loin du rivage (7,5 \% de M.L.) et les autres échantillons recueillis sur la partie haute de l'estran (teneur moyenne (t.m.) $=20,2 \%$ M.L.). Au Pouldu, les concentrations les plus importantes sont situées dans la partie nord-est de la plage bien exposée aux houles du large. A Aod Vraz, les dépôts sont essentiellement localisés dans la partie sud-ouest de la plage, pour la même raison; dans la partie septentrionale de la même plage, relativement abritée, les concentrations sont absentes ou extrêmement faibles $(0,3 \%$ de M.L. pour le prélèvement 14). Plusieurs processus peuvent interférer dans le tri sélectif des M.L.A Aod Vraz, la dérive littorale en direction du nord-est (avec entraînement de minéraux légers) se superpose à l'action des vagues surtout efficace dans la partie sud-ouest de la plage.

Les concentrations sont parfois plus élevées dans les petites criques jouant le rôle de pièges que sur les grandes plages dunaires largement ouvertes (à l'îlc de Batz, net contraste entre la crique près Kerabandu ( $58,2 \%$ de M.L.) et la plage d'Aod Wenn (9,9\%), sans qu'il y ait cependant de règle générale comme l'indiquent les variations sur la plage 
dunaire du Pouldu $(11,7$ à $55,8 \%)$. Localement, les teneurs les plus fortes coïncident avec la prédominance de M.L. très denses (Kerezeant avec plus de $80 \%$ d'ilménite) ; inversement, les forts pourcentages en minéraux " légers ", comme l'amphibole, se traduisent souvent par une faible concentration totale en M.L. (prélèvement 32 dans l'Aber, avec plus de $85 \%$ d'amphibole).

Les accumulations de M.L. peuvent être temporaires et liées aux périodes d'amaigrissement de la plage, avec éloignement sélectif des minéraux légers. Les variations dans l'amplitude des marées commandent la largeur de l'estran soumise à l'action des vagues. Par temps calme, le triage hydraulique est peu poussé et n'affecte qu'une épaisseur réduite de sable, d'où la faible puissance des occurrences. Lors des périodes de tempêtes, quand la direction des vents coïncide avec un fetch important (vents de secteur W à NW), la masse de sable mise en mouvement et le triage différentiel augmentent considérablement, d'où l'importance des dépôts.

La contamination des sables terrigènes par des sables organogènes entraîne un appauvrissement relatif du potentiel sableux en M.L. Les teneurs maximales au $\mathrm{Co}^{3} \mathrm{Ca}$ (toujours $>50 \%$, max. : $82 \%$ ) sont localisées aux environs de Santec (estran, dunes, zone infratidale) (Boillot, 1961, 1964 ; Conversey, 1962), dans une zone exposée aux fortes houles d'ouest. Les teneurs sont, au contraire, très faibles (le plus souvent, t.m. de l'ordre de 2 à $3 \%$ ) sur la côte ouest de la baie de Morlaix, beaucoup plus abritée (Masson, 1969 ; Loarer, 1975 ; M.M. Chauris, inédit) ; l'Aber de Roscoff peut également être rattaché à ce secteur (Francis-Bœuf et al., 1949). Les teneurs sont intermédiaires à l'île de Batz. (Les dunes du sud-ouest de l'île, à teneur élevée, pourraient représenter un témoin isolé du manteau dunaire de Santec). Le caractère très organogène des sables de la plage du Dossen peut expliquer l'absence générale de concentrations de M.L. sur l'estran. Par suite de très faibles teneurs en M.L. disséminés, less conditions hydrodynamiques nécessaires au triage ne peuvent apparaître ici que d'une manière exceptionnelle.

\section{III. - REPARTITION DES DIFFÉRENTS MINÉRAUX LOURDS.}

60 analyses ont été effectuées sur les concentrations de M.L. Selon les prélèvements, 13 à 19 M.L. ont été déterminés ; le plus souvent, les occurrences renferment 16 à 17 espèces différentes. Le calcul des moyennes montre que le spectre régional est dominé par quelques minéraux : amphibole, grenat, ilménite et tourmaline : à elles seules, ces 4 espèces constituent plus de $85 \%$ des M.L. Parmi les autres minéraux décelés avec une certaine abondance, on note le sphène, la staurotide et l'épidote ; puis, avec des teneurs $<1 \%$ (sauf quelques exceptions remarquables) magnétite, monazite, zircon et rutile. D'autres minéraux n'apparaissent généralement qu'à l'état de traces : c'est le cas des silicates d'alumine (disthène, sillimanite, andalousite), de l'apatite, de l'anatase et du corindon. Cassitérite, scheelite et pyrite sont nettement plus sporadiques. Enfin, quelques minéraux sont extrêmement rares : marcasite, spinelle, xénotime. 
AMPHIBOLE (fig. 2)

L'amphibole (essentiellement hornblende verte) est le minéral lourd le plus répandu $(\sim 34 \%)$. Ce pourcentage élevé souligne le caractère immature des concentrations. L'amphibole est observée dans tous les prélèvements, mais avec des teneurs extrêmement variables (de l'ordre de $1 \%$ à plus de $80 \%$ ). Les teneurs très faibles sont essentiellement localisées à l'extrémité occidentale de la région. Mis à part quelques prélèvements où l'amphibole est manifestement en relation avec les roches-mères voisines (diorites de Creac'h André, amphibolites de Tregondern...), les pourcentages élevés semblent beaucoup plus refléter les processus de triage différentiel (facilités par la forme oblongue et la densité relativement faible du minéral) qu'une source proximale, comme le suggère la coïncidence fréquente des hautes teneurs avec les zones de " basse énergie » pauvres en M.L. : prélèvements 23 et 32 , respectivement $73,8 \%$ et $86,1 \%$ d'amphibole, $4 \%$ et $7,5 \%$ de M.L. L'exemple le plus significatif est fourni par deux prélèvements voisins effectués à Aod Vraz, l'un (13) sur la partie supérieure de l'estran (amphibole à l'état de traces, $55 \%$ de M.L.), l'autre (12), plus bas sur l'estran (54,9\% d'amphibole, $7,2 \%$ de M.L.).

L'aptitude de l'amphibole à rester longtemps en suspension rend souvent la recherche des sources aléatoires. Dans la région de Roscoff, les roches-mères possibles (amphibolites de la Penzé et minces lits intragneissiques ; diorites de l'île de Batz et de Créac'h André) sont relativement subordonnées et ne peuvent, malgré une influence locale évidente, constituer des sources majeures. L'émoussé plus ou moins accusé de l'amphibole dans un certain nombre de prélèvements, va également dans le sens d'une origine en partie distale. A priori, l'amphibole pourrait dériver pro parte du stock de M.L. du cortège de la Manche (Duplaix el al., 1968 ; Giresse et al., 1970 ; Larsonneur et al., 1982). Toutefois cette interprétation n'explique pas pourquoi les teneurs deviennent faibles $(<10 \%$ et plus souvent $<1 \%)$ à l'ouest de la région étudiée (à partir de l'île de Siec) dans le reste du Pays de Léon (Chauris, 1982 et travaux en cours). Un apport significatif pourrait être fourni par les roches basiques du Trégorrois situé à l'est de la baie de Morlaix (Chauris, 1985).

\section{GRENAT (fig. 3)}

Avec un pourcentage moyen d'environ $26 \%$, le grenat occupe la deuxième place parmi les M.L. Les tencurs oscillent entre deux extrêmes ( 1 à $2 \%$ à Tregondern, plus de $95 \%$ à Siec). Du fait de sa morphologie et de sa densité, le grenat offre un comportement hydraulique différent de celui de l'amphibole et se concentre préférentiellement dans les zones de haute énergie. La comparaison des deux prélèvements voisins d'Aod Vraz est très significative : 13, partie supérieure de l'estran (haute énergie) : grenat $68,2 \%$; amphibole à l'état de traces ; 12 , partie plus basse de l'estran (énergie plus faible) : grenat à l'état de traces, amphibole : $54,9 \%$. 

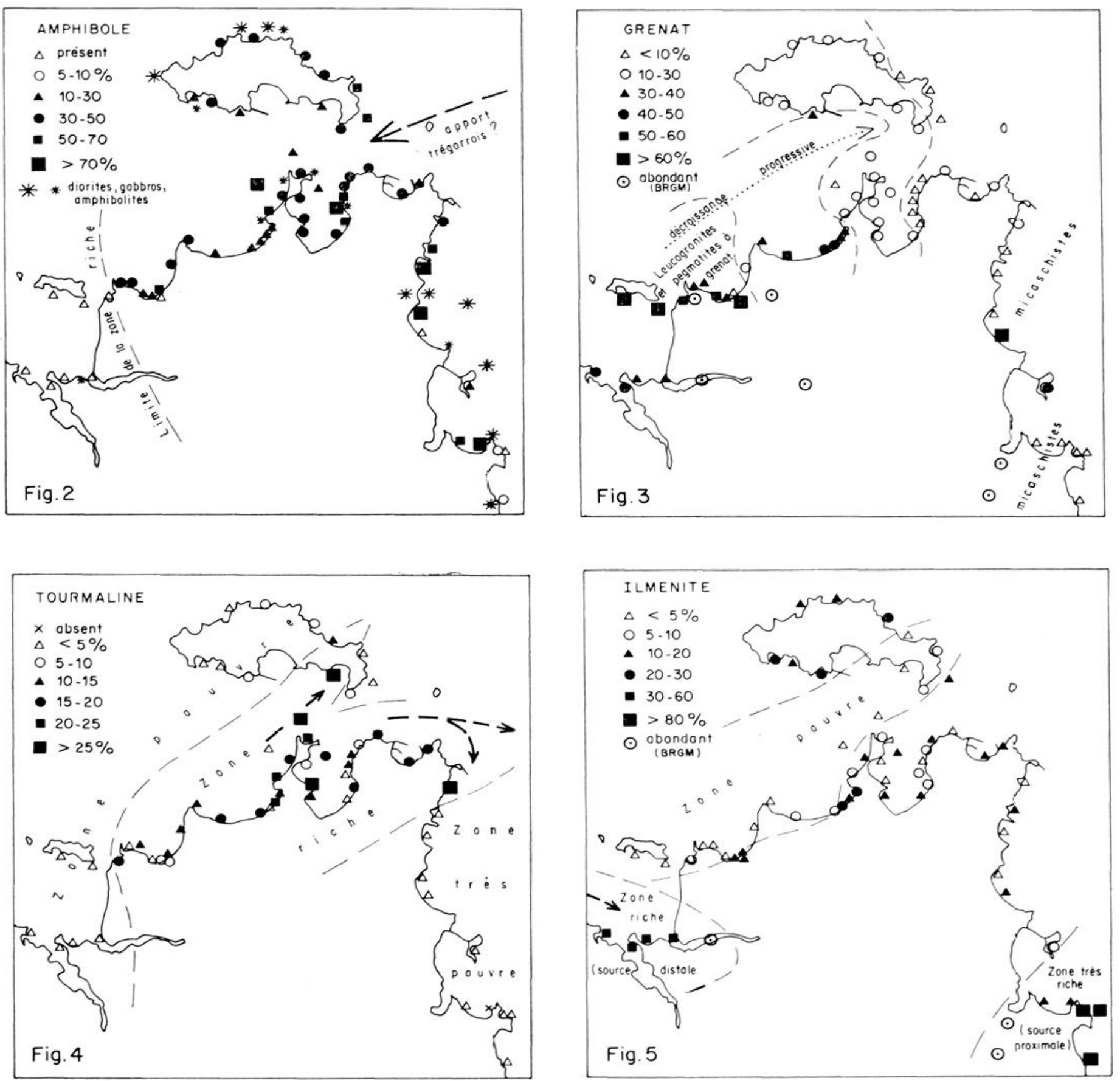

FIG. 2 à 11 - Tirets = limite approximative des zones. Les flèches indiquent la direction estimée du déplacement. Pourcentage des minéraux lourds en poids.

FIG. 2 to 11 - Dasheb line = approximative boundary of zones. Arrows show estimated direction of transfer. Percentage of heavy minerals in weight. 
L'examen de la carte de répartition souligne à l'évidence le rôle majeur joué par les roches-mères d'origine locale. Les leucogranites et les pegmatites à grenat, si fréquents dans les environs de Siec-Santec constituent un premier exemple. A leur proximité, les teneurs sont extrêmement élevées ; elles décroissent progressivement en direction de l'est (possibilité de tracer des courbes d'iso-teneurs). La diminution est même sensible à l'échelle d'une seule grande plage (éch. 16 à 20 au Pouldu) ou d'une anse (opposition entre les rives ouest et est de l'Aber); elle est nette également dans la partic est de l'île de Batz. Les micaschistes de la partie occidentale de la baix de Morlaix forment une seconde rochemère dont l'impact est toutefois moins prononcé. L'origine proximale dominante est également confirméc par la morphoscopie des grenats, souvent parfaitement automorphes ou en esquilles aux arêtes vives. L'influence d'une éventuelle source distale paraît ici très accessoire.

\section{TOURMALINE (fig. 4)}

La teneur-moyenne est de l'ordre de $10 \%$; les pourcentages varient d'une fraction de $\%$ à plus de $40 \%$. La carte de répartition montre la présence d'une zone riche isolant deux zones pauvres à très pauvres. La coïncidence, en première approximation, de la zone riche avec un grand développement des leucogranites et des pegmatites à tourmaline témoigne d'une origine proximale, confirmće par la morphoscopie. Dans le détail, un décalage vers l'est apparaît entre le domaine principal d'affleurements des roches tourmalinifères et la zone des sables riches en tourmaline. Cette constatation suggère une migration de la tourmaline d'ouest en est, nettement plus prononcée que dans le cas du grenat. Ce transit paraît se poursuivre jusque sur la côte du Petit-Trégor (Chauris, 1984).

\section{ILMÉNITE (fig. 5)}

Les teneurs en ilménite vont de moins de $1 \%$ à plus de $85 \%$. La teneur moyenne (t.m.) - de l'ordre de $17 \%$ - s'avère assez peu significative du fait de la localisation des teneurs élevées essentiellement dans les zones ouest (Quilliec, t.m. $=44,1 \%$ ) et est (Penzé t.m. = 83,8 \%). Ces deux districts mis à part, les teneurs sont relativement faibles (toujours $<30 \%$, avec une moyenne $<10 \%$ ). Un domaine particulièrement pauvre prend en écharpe la région entre l'île de Siec et l'est de l'île de Batz. Les zones riches du Quilliec et de la Penzé se distinguent par leur tencur (du simple au double), leur granulométrie (fine à l'ouest, grossière à l'est) et leur morphologie (souvent bien émoussée à l'ouest, très anguleuse à l'est). Cette opposition traduit deux sources différentes : à l'est, une origine proximale, en relation avec les amphibolites de la Penzé ; à l'ouest, une origine distale, incléterminée, appuyée également par la présence en pourcentages significatifs de minéraux résistants (zircon, rutile, monazite).

SPHËNE (fIg. 6)

La reconnaissance précise de sources primaires localisées (essentielle- 
ment les diorites de l'île de Batz) fait de ce minéral un excellent traceur. Son abondance dans certaines concentrations de l'île s'avère tout à fait exceptionnelle en Bretagne : la teneur moyenne des 11 échantillons est ici de $12,4 \%$.

A partir des affleurements de cliorites, situés dans les parties septentrionale et occidentale de l'île, il est possible de suivre la migration du sphène en direction de l'est. Le prélèvement le plus riche $(26,5 \%)$ coïncide avec le plus large affleurement de diorite. Vers l'est, les teneurs décroissent, mais de manière irrégulière : sur la côte nord et sur la côte sud, les pourcentages accusent un "creux "vers la partie médiane de l'île (relativement à l'abri de la dérive littorale vers l'est) pour augmenter à nouvcau vers l'est. Il est également possible de préciser les déplacements vers les rives de la presqu'île roscovite. La faiblesse des teneurs à l'ouest de Corn al Loa indique que le transit se fait en direction du sud-est et de l'est. Les migrations vers et dans l'Aber de Roscoff montrent une décroissance d'ensemble avec l'éloignement de l'île de Batz. Au fond du port de Roscoff (37), les teneurs sont encore de $5 \%$; plus à l'est, elles chutent brusquement et le sphène n'est plus présent qu'à l'état de traces, voire même absent ; seul un petit apport localisé est en relation avec les diorites de Créac'h André.

\section{STAUROTIDE (fig. 7)}

La staurotide est omniprésente (59/60 éch.), mais avec des teneurs variables, souvent de l'ordre de 1 à $2 \%$ ou même inférieures ; les teneurs supérieures à $5 \%$ restent exceptionnelles. Dans le cas des faibles teneurs, une partie de la staurotide peut représenter l'étalement du "fond régional " caractéristique de certains domaines cristallophylliens, comme le Pays de Léon (Chauris, 1982); dans les domaines dépourvus de telles formations, comme dans le Petit-Trégor, les teneurs sont généralement très faibles, voire inexistantes (Chauris, 1985). La côte nord-ouest de la baie de Morlaix se distingue nettement du fond régional. La forte t.m. de ce secteur $(11,8 \%)$ est en relation proximale avec un complexe micaschisteux, localement très riche en staurotide, en grande partie immergé (Chauris, inédit). Les teneurs observées sur les plages de Roscoff nord (t.m. $=3,8 \%$ ), sur la rive occidentale de l'Aber $(\mathrm{t} . \mathrm{m} .=4,3 \%)$ et sur quelques plages orientales de Batz sont peut-être en relation avec la même source. La forte tencur ( $>10 \%$ ) notée près de Siec pourrait refléter l'extension vers le nord-ouest des septa micaschisteux découverts au sud de Santec.

\section{SILICATES D'ALUMINE ET CORINDON.}

Ces minéraux sont toujours observés en traces. Le disthène est omniprésent $(58 / 60)$. Son origine est probablement distale (absence de formations à disthène aux environs de Roscoff). La sillimanite a été observée dans 30 éch./60. La division de la région étudiée par une ligne nord-sud port de Batz--Perharidic fait apparaître deux domaines différents : une zone ouest où la sillimanite est rare $(7 / 27)$ et une zone est où elle est fréquente (23/33). La même disposition qui se retrouve également pour l'andalousite (respectivement 18,27 et $31 / 33$ ) et pour le corindon (14/27 

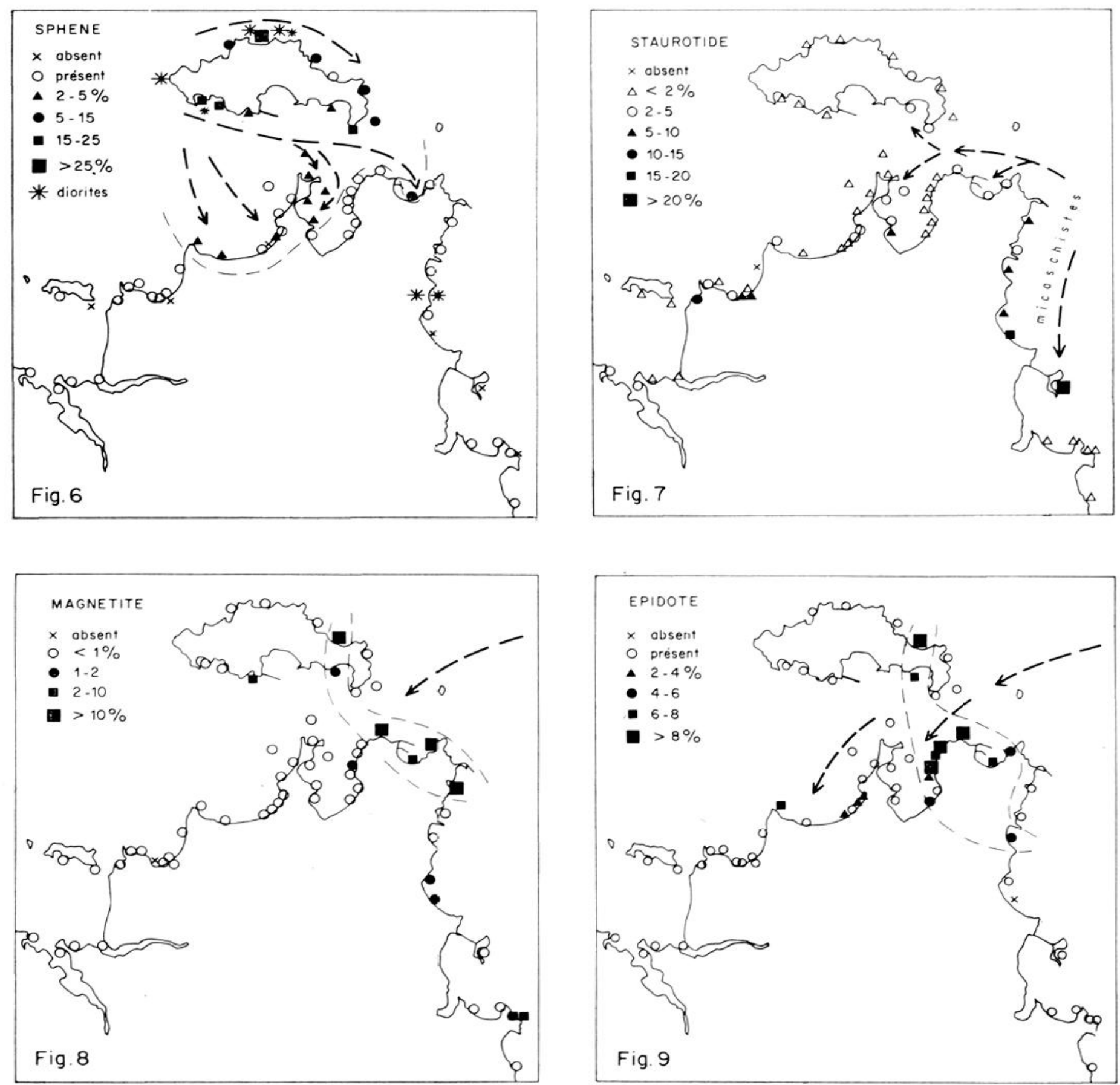

et $26 / 33$ ) ne semble pas aléatoire. L'hypothèse d'une zonéographie métamorphique pourrait peut-être s'appliquer à la sillimanite qui disparaît totalement du district de la Penzé et de son prolongement en baie de Morlaix (Chauris, 1984). L'appel à des apports distaux n'explique pas pourquoi la grande plage du Pouldu et ses abords sont totalement dépourvus de sillimanite. L'hypothèse d'un triage différentiel entre une côte occidentale exposée aux grandes houles de l'ouest et une côte orientale plus abritée, expliquerait qu'andalousite et sillimanite, relativement légères, se déposent dans les zones plus calmes (Aber, baie de Morlaix). Le cas du corindon reste énigmatique. 
MAGNÉTITE ET EPIDOTE (fig. 8 et 9)

La magnétite est omniprésente (59/60), mais le plus souvent avec des teneurs $<1 \%$. Mises à part quelques faibles concentrations sporadiques (de l'ordre de $2 \%$ ) (Tregondern...), la magnétite est localisée aux environs immédiats de Roscoff ( 3 prélèvements $>10 \%$ ) et vers l'est de l'île de Batz (1 prélèvement $>10 \%$ ). L'épidote est également omniprésente $(59 / 60)$, mais généralement avec des pourcentages très faibles $(<2 \%)$. Une zone de net enrichissement apparaît à Roscoff et vers l'est de l'île de Batz (jusqu'à $16 \%$ ).

Une coïncidence approximative se manifeste ainsi dans la répartition de ces deux espèces, avec toutefois une extension plus prononcée vers l'ouest pour l'épidote (rive est de l'Aber, plage du Pouldu). Les recherches géologiques n'ont pas permis de localiser une source proximale de ces minéraux. Un mince lit riche en épidote a été signalé à Perharidic (Sandréa, 1958), mais son extension très réduite et l'absence au moins apparente de magnétite suggèrent une influence négligeable. Les occurrences observées pourraient être rattachées à la présence présumée d'un panneau, actuellement ennoyé, de schistes verts (épidotite à magnétite) dans le prolongement occidental des complexes méta-volcaniques du Trégor où ces formations se traduisent par l'apparition sur l'estran de sables fortement enrichis en épidote et en magnétite (Porz Mellec en Plestin, Chauris, inédit).

ZIRCON - RUTILE - MONAZITE - XÉNOTIME (fig. 10)

Les teneurs en zircon sont généralement faibles et $<1 \%$. Toutefois, dans le district du Quilliec, la teneur moyenne s'élève à $3,1 \%$. Les pourcentages en rutile sont encore plus bas et, en règle générale, nettement $<$ à $1 \%$. Ici aussi, le district du Quilliec se singularise par quelques teneurs plus fortes (jusqu'à $2,1 \%$ ). La monazite est plus répandue ; cependant, mis à part 4 prélèvements dispersés, seul le district du Quilliec montre dans tous les échantillons des pourcentages $>$ à $2 \%$ (t.m. $=$ $2,8 \%)$. Au Quilliec, la somme des 3 minéraux donne une t.m. de 6,9\%. L'origine de ces minéraux résistants est difficile à préciser. Leur concentration préférentielle dans le district dunaire du Quilliec et, dans une moindre mesure sur les plages dunaires d'Aod Vraz et du Pouldu, suggère une origine primaire distale. Le xénotime est extrêmement rare et n'a été décelé que dans les sables grenatifères de Siec.

\section{CASSITÉRITE — SCHEELITE — APATITE (fig. 11)}

Dix-sept échantillons sur soixante ont montré de la cassitérite sous forme de microtraces à grain fin, de teinte brunâtre. Treize échantillons sur soixante ont révélé des microtraces de scheelite. Il n'apparaît pas de relation directe entre les deux minéraux : 7 prélèvements renferment à la fois cassitérite et scheelite. La région étudiée peut être divisée en deux parties : à l'ouest, un secteur surtout caractérisé par la cassitérite (12 éch.) et la rareté de la scheelite (2 éch.) ; à l'cst, inversement un secteur à scheelite dominante $(11$ éch.) et à cassitérite subordonnée (5 éch.). A priori, la finesse et l'émoussé de la cassitérite peuvent indiquer 

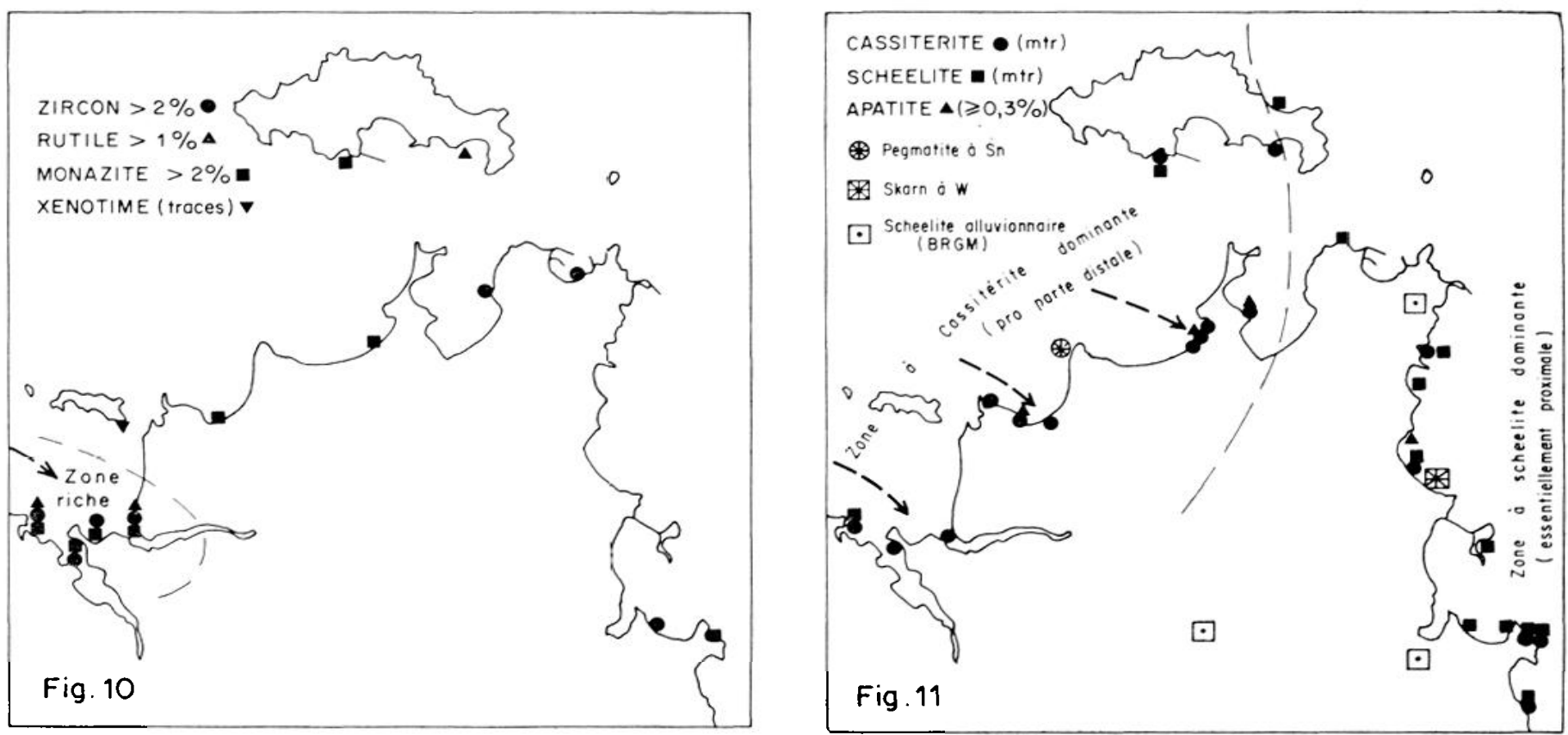

une provenance relativement lointaine, reflet du cachet stannifère du Pays de Léon, en relation avec le vaste pluton de Saint-Renan. Le caractère distal peut être également appuyé par la localisation préférentielle de la cassitérite sur les plages dunaires des districts du Quilliec, d'Aod Vraz et du Pouldu. Il importe toutefois de remarquer que la cassitérite a été découverte en place dans une pegmatite sodo-lithique à l'ouest de Corn al Loa (Chauris et al., 1981) et que des teneurs relativement élevées en étain (jusqu'à $160 \mathrm{gr} / \mathrm{t}$ ) ont été décelées dans des aplites de Santec. La cassitérite des estrans de la baie de Morlaix est, selon toute probabilité, en relation avec le granite stannilère voisin de Carantec (Chauris, 1975). La scheelite, plus fragile que la cassitérite, paraît avoir une origine essentiellement proximale. Elle pourrait provenir des skarns découverts dans la région (Chauris et al., 1978b ; Chauris, inédit) ; les prospections alluvionnaires du B.R.G.M. suggèrent la présence d'autres occurrences. L'apatite est omniprésente $(56 / 60)$, mais presque toujours en traces. Les teneurs d'Aod Vraz, du Pouldu, de l'Aber et de Créac'h André sont, selon toute probabilité, en relation avec les pegmatites voisines, parfois riches en apatite (Chauris, 1978a).

\section{IV. - RESULTATS GÉOMORPHOLOGIQUES.}

A l'issue de l'étude systématique des différents minéraux lourds de la région de Roscoff, il est possible de reconnaître une succession de petits districts juxtaposés. Ces districts se distinguent les uns des autres par la surabondance ou, au contraire, la rareté de quelques espèces principales du spectre moyen régional et, parfois par la teneur élevée d'espèces ailleurs toujours subordonnées. Des transitions peuvent apparaître entre des districts voisins. 
En première approximation, les deux principaux types de côtes du littoral contraposé de Roscoff (rivages rochcux ourlés de petites criques et rivages dunaires bordés de grandes plages) contrôlent d'une manière différente l'alimentation des plages en sable et en conséquence l'origine des M.L. qui forment localement de petits placers sur les estrans; l'anse de l'Aber constitue un cas un peu particulier.

- Les plages des zones rocheuses offrent fréquemment une importante alimentation proximale. Les vagues trient les M.L. disséminés dans les arènes et le head qui empâtent les monadnocks. Entre les pointes rocheuses, les criques s'approfondissent par déblayage de ces formations meubles soumises à un intense lessivage. Les dépôts constituent de petites " cellules " plus ou moins différenciées les unes des autres qui reflètent, au moins en partie, la nature variée et le potentiel plus ou moins élevé des arènes et du head en M.L. Parfois, les concentrations sont presque monominérales : grenat de Siec (leucogranite), ilménite de la Penzé (amphibolites)... ou sont très riches en un minéral rare ailleurs (sphène des diorites de Batz). L'érosion provoque une introduction constante de matériaux frais. L'examen morphoscopique montre des minéraux très anguleux qui trahissent la jeunesse des accumulations. Il n'y a pas de formations-relais entre les altérites et les accumulations de M.L. Les petites criques jouent le rôle de pièges et les concentrations en M.L. augmentent progressivement. Dans le cas d'exposition favorable, des apports distaux plus ou moins importants ne sont évidemment pas à exclure.

- Les plages adossées aux dunes reprennent par érosion le sable d'origine distale accumulé dans les cordons dunaires. Les plages bordières reflètent ainsi essentiellement des apports lointains. Il devient alors illusoire de rechercher d'une manière précise la source des M.L. Les dunes jouent le rôle de formations-relais. La source primaire des M.L. disséminés dans les dunes est distale, mais l'origine secondaire des M.L. concentrés sur les plages dunaires est, en fait, proximale. Sur les plages dunaires, le spectre minéralogique est plus "étalé " que sur les plages des zones rocheuses et plus comparable d'une plage à l'autre, par suite d'une certaine homogénéisation à partir de sources multiples et lointaines. Certains M.L. sont fortement émoussés. Toutefois, des apports en provenance des zones rocheuses voisines ne sont évidemment pas à exclure ; le transit dû à la dérive littorale provoque l'interférence des sources et une contamination plus ou moins importante. Les sables dunaires de Santec (Duplaix et al., 1968)) sont pauvres en M.L. ( $\sim 0,4 \%$ seulement). Les trois-quarts du spectre sont représentés par l'amphibole, le grenat et la tourmaline en proportion à peu près égale (l'ilménite n'a pas été comptabilisée). A ces espèces s'ajoutent épidote, staurotide, sphène, zircon, rutile, monazite, anatase, andalousite... Les résultats des prospections alluvionnaires du B.R.G.M. (Guigues et al., 1969 ; atlas inédits) précisent la composition minéralogique des sables dunaires drainés par les petits ruisseaux de la région. Les six échantillons examinés renferment amphibole, grenat, ilménite, tourmaline, staurotide, épidote, magnétite, monazite, zircon, rutile et anatase; les autres M.L. sont plus sporadiques : disthène et cassitérite $(4 ; 6)$, corindon $(2 / 6)$, andalousite et xénotime $(1 / 6)$. Le spectre minćralogique des dunes apparaît ainsi très comparable à celui des plages bordières. Dans les zones à faible énergie, il n'y a pas 
d'enrichissement (à l'ouest de Corn al Loa, le pourcentage en M.L. sur l'estran est de l'ordre de $0,3 \%$ ). Dans les zones à haute énergie, le facteur de concentration peut dépasser 100 fois.

- Les grèves de l'anse de l'Aber revêtent un intérêt particulier du fait qu'elles résultent de l'intervention récente de la mer dans un ancien marais littoral. Le contraste minéralogique entre la rive ouest et est de l'anse suggère la complexité des apports et des remaniements. La plus grande partie des sables provient de l'érosion éolienne des dunes situées immédiatement à l'ouest (Pouldu et Perharidic), sous l'influence des vents dominants. Cette origine proximale est confirmée par la comparaison des cortèges des principaux M.L. (amphibole, grenat, tourmaline, ilménite). Les différences de pourcentage proviennent essentiellement des triages différentiels dus aux conditions d'énergie différentes entre les plages dunaires occidentales exposées et les grèves abritées de l'Aber (diminution des pourcentages en grenat, augmentation des pourcentages en amphibole). Une autre partie des sables de l'Aber pénètre dans l'anse par le nord, en particulier sous l'influence des houles du NNE (direction du seul fetch influent). La migration des sables vers la partie sud de l'anse est matérialisée par le profil dissymétrique de plusieurs plages et de petites dunes hydrauliques. Les apports sableux en provenance du nord ou de l'est sont appuyés également par différents M.L. (sphène, épidote, staurotide).

\section{CONCLUSION.}

L'étude des accumulations de minéraux lourds peut contribuer à préciser la dynamique des dépôts sableux dans une région à morphologie littorale aussi variée que celle présentée par la côte contraposée de Roscoff. La superposition des cartes de teneurs des différents minéraux (fig. 2 à 11) souligne la complexité des transits qui peuvent même s'effectuer en sens opposés. Sur une dérive générale d'ouest en est (établie par le grenat et la tourmaline) peuvent interférer des déplacements du nord au sud (prouvés par le sphène) et probablement d'est en ouest (suggérés par l'amphibole, l'épidote, la magnétite et peut-être la staurotide). Quelques minéraux permettent de tracer, avec une certaine précision, des halos de dispersion (avec parfois [grenat] des courbes d'isoteneurs) à partir de roches-mères favorables (amphibolites, micaschistes, diorites, leucogranites). De nombreux minéraux lourds dérivent sans formation-relais des altérites qui jouent le rôle de sources primaires souvent proximales. D'autres minéraux lourds proviennent des sables dunaires qui forment des relais secondaires : ici la source primaire, imprécisée, est toujours distale. Le triage différentiel contribue à masquer les filiations. Il peut parfois être extrêmement poussé : dans les petits placers de Siec (éch. 5 et 6 ), la teneur en grenat est $\sim 95 \%$, le pourcentage en tourmaline $<1 \%$, alors que ces deux minéraux sont abondants dans les leucogranites voisins. Un triage sélectif aussi intense est à relier aux différences de densité et de morphologie entre les deux minéraux. Dans l'ensemble, les concentrations offrent un net indice de jeunesse, attesté par le pourcentage élevé en minéraux anguleux et souvent par les fortes teneurs en amphibole. Le spectre d'ensemble des minéraux lourds est ici typiquement immature. La tourmaline, minéral très résistant, est nor- 
malement comptabilisé dans l'indice ZTR (zircon + tourmaline + rutile) pour quantifier le degré de maturité des sables. Il apparaît toutefois évident par l'exemple de la région de Roscoff que cet indice peut être totalement dépourvu de signification dans le cas de districts où des roches très riches en tourmaline constituent une source surabondante de ce minéral.

Remerciements. - Nous remercions vivement Y. Le Fur, Chef de la Division Minière B.R.G.M. du Massif Armoricain, qui nous a permis de consulter les admirables atlas inédits des prospections alluvionnaires en lit vif ; H. Chevance, Chef du Laboratoire de Minéralogie du B.R.G.M. à Nantes qui a analysé les concentrés de minéraux lourds ; J.L. Travers qui a redessiné les cartes et $\mathrm{D}$. Rachebœuf qui a dactylographié le manuscrit. Nous témoignons notre profonde reconnaissance à $M$. le Professcur $A$. Guilcher pour ses remarques et suggestions. Ce travail a été entrepris dans le cadre du G.I.S. 410012 «Ocćanologie et Géodynamique ».

\section{REFERENCES BIBLIOGRAPHIQUES}

Auffret G.A. et Douvilt.e J.L. (1974). Observations sur la dynamique des sables de la Pierre Noire (baie de Morlaix, Finistère, France). Bull. B.R.G.M. $2^{\mathrm{e}}$ sér., sect. IV, 1, p. 5-18, 8 fig., 2 tabl., 1 pl.

Barrois C. (1909). Carte géologique au 1/80 000, feuille Lannion.

Boillot G. (1961). La répartition des sédiments en baie de Morlaix et en baie de Siec. Cahiers de Biologie Marine, II, p. 53-66, 8 fig., 1 pl.

Bonllot G. (1964). Géologie de la Manche occidentale. Ann. Institut Océanogr., XLII, 220 p.

Bourcart J. (1948). Le Quaternaire des grèves de Roscoff (Finistère). Bull. Soc. Géo!'. Fr., 5, XVIII, p. 181-197, 3 fig.

Burns V.M. (1979). Marine placer minerals. In vol. 6 " Marine Minerals ». Reviews in Mineralogy, Mineral. Soc. America, p. 347-380.

Castro C. (1981). Les dunes du Léon et les problèmes de leur conservation. Thèse de $3^{c}$ cycle, Univ. Bretagne Occidentale, Brest, 176 p.

Chasse C. (1972). Economie sédimentaire et biologique des estrans meubles des côtes de Bretagne. Thèse d'Etat, Sciences Nat., Univ. Paris VI. 293 p.

Chauris L. (1975). Minéralisations stanno-wolframifères dans le granite de Carantec (Bretagne). C.R. Acad. Sci., Paris, sér. D, t. 280, p. 2421-2424, 1 fig.

Chauris L. (1982). A la recherche des placers littoraux : concentrations de sables lourds sur les plages du Nord-Finistère (Massif armoricain). 107e Congr. Nat. Soc. Sav., Brest, Sciences, fasc. III, p. 119-130, 1 fig.. 2 tabl., 1 pl.

Chauris L. (1984). Concentration de sables lourds sur les plages de la baie de Morlaix (Finistère). Norois, t. 31, no 123. p. 407-426, 1 fig., 2 tabl.

Chauris L. (1985). Minéraux lourds des plages et arrière-pays : le cas du Petit-Trégor (Massif armoricain). 110 Congr. Nat. Soc. Sav., Montpellier, sect. Sciences, fasc. 1, p. 183-194, 9 fig., 2 tabl.

Chauris L. et Corre Y. (1978a). Une pegmatite à gros béryls sur l'estran de l'île de Batz (Finistère, France). Bull. Minéral., 101, p. 575.

Chauris L. et Corre Y. (1978b). Skarn à idocrase, grossulaire et scheelite de Plougoulm (Finistère, France). Bull. Minéral., 101, p. 576-577, 1 fig., 1 tabl.

Chauris L., Corre Y. et Cotten J. (1981). Une pegmatite à tourmaline lithique dans le massif granitique de Roscoff (Finistère, France). C.R. Acad. Sci., Paris, t. 293, sér. II, p. 601-604.

Conversey R. (1962). La côte nord de la Bretagne, de Guissény' à Saint-Pol-de-Léon. D.E.S. de Géographic physique. Univ. de Nancy, inédit.

Cronan D.S. (1980). Underwater minerals. Academic Press, London, 362 p.

Croguennoc M.P. (1981). Sites du Pouldu et du Dossen, Santec. Rapports inédits de l'Institut de Géogr. de l'Université de Brest. 
Devismes P. (1978). Atlas photographique des minéraux d'alluvions Mém. B.R.G.M., $\mathrm{n}^{\circ} 95,206 \mathrm{p}$.

Dupeuble P.A. (1964). Les minéraux lourds de quelques sédiments actuels de la région de Roscoff. C.R. Somm. Soc. Géol. Fr., p. 260-261.

Duplaix S. (1946). Les minéraux lourds de quelques sables du Finistère. C.R. Somm. Soc. Géol. Fr., p. 131-133.

Duplaix S. et Boillot G. (1968). Sur la minéralogie et l'origine des sables siliceux contenus dans les sédiments de la Manche occidentale. Rev. Géogr. Phys. et Géol. Dynam. (2), X, fasc. 2, p. 147-161, 3 fig., 7 tabl.

Francis-Bgelf C., Bourcart J. et Duplaix S. (1949). La couverture sédimentaire de l'Aber de Roscoff (Finistère). Sédiment. et Quaternaire, Led-Sam édit., Bordeaux, p. 21-48, 7 fig., 6 tabl.

Giresse P. et Larsonneur C. (1970). La succession des apports sableux de la Manche centrale grâce à l'étude des minéraux lourds. Rev. Géogr. Phys. et Géol. Dynam. (2), XII, fasc. 1, p. 41-62, 8 fig., 1 tabl.

Guigues J. et Devismes P. (1969). La prospection minière à la batée dans le Massif armoricain. Mém. B.R.G.M., $\mathrm{n}^{\circ} 71,172$ p.

Guilcher A. (1954). Morphologie littorale et sous-marine. Presses Univers. Fr., Paris, $214 \mathrm{p}$.

Hallegouet B. (1978). L'évolution des massifs dunaires du Pays de Léon. Penn ar Bed, Brest, 11, 95, p. 417-430, 9 fig.

Hallegouet B. (1981). Les crêtes littorales dunifiées du Massif armoricain, France : formation et évolution. Géogr. Phys, et Quaternaire, 35, 2, p. 205-218, 15 fig.

Larsonneur C., Bouysse P. et Auffret J.P. (1982). The superficial sediments of the English Channel and its Western Approaches. Sedimentology, 29, p. 851-864, 4 fig.

LE Gorgeu J.P. et Boillot G. (1964). La migration des sables marqués au large de Roscoff (Finistère). C.R. Acad. Sci., Paris, 259, p. 4082-4085.

LoArer R. (1975). Ile Callot. Morphologie et sédimentologie. Mém. Maîtrise Géographie, Université de Brest, 126 p. +73 p. (annexes).

Macdonald E.H. (1983). Alluvial mining. Chapman and Hall, London, 508 p.

Masson M. (1969). Le Graoli sur la côte de Saint-Pol-de-Léon. Sédimentologie et cartographie. Mém. de Maîtrise de Géographie, Université de Brest, 62 p.

Morzadec-Kerfourn M.T. (1974). Variations de la ligne de rivage armoricaine au Quaternaire. Analyses polliniques de dépôts organiques littoraux. Mém. Soc. Géol. Minéral. Bretagne, 17, 208 p.

SANDrea A. (1958). Contribution à la lithologie de la côte nord de la Bretagne : de l'île de Siec à la baic de Perros. Bull. Service Carte Géol. Fr., LVI, 258, p. 505-616. 